\title{
Machine Learning for Communication Performance Enhancement
}

\author{
Xin-Lin Huang $\mathbb{D}^{1},{ }^{1}$ Fei Hu, ${ }^{2}$ Xiaomin Ma $\left(\mathbb{D},{ }^{3}\right.$ Ioannis Krikidis, ${ }^{4}$ and Dejan Vukobratovic ${ }^{5}$ \\ ${ }^{1}$ Tongji University, Shanghai, China \\ ${ }^{2}$ The University of Alabama, Tuscaloosa, AL, USA \\ ${ }^{3}$ Oral Roberts University, Tulsa, OK, USA \\ ${ }^{4}$ University of Cyprus, Nicosia, Cyprus \\ ${ }^{5}$ University of Novi Sad, Novi Sad, Serbia
}

Correspondence should be addressed to Xin-Lin Huang; xlhuang@tongji.edu.cn

Received 8 October 2018; Accepted 9 October 2018; Published 1 November 2018

Copyright (C) 2018 Xin-Lin Huang et al. This is an open access article distributed under the Creative Commons Attribution License, which permits unrestricted use, distribution, and reproduction in any medium, provided the original work is properly cited.

Along with the fast development of mobile communication technologies, a large number of high-quality wireless services are required. According to the report of Cisco VNI Global Mobile Data Traffic Forecast 2017, global mobile data traffic will increase nearly sevenfold from 2016 to 2021, and mobile network connection speeds will increase threefold by 2021 . There is a big gap between the future requirements of wireless services and current communications technologies, even using $4 \mathrm{G} / 5 \mathrm{G}$ technique. How to design intelligent algorithms/schemes to make full use of the limited wireless resources is the theme of this special issue. As an important discipline, machine learning includes pattern recognition and computational learning theory in artificial intelligence, and algorithms to learn from the past and make predictions in complicated scenarios. It can be used to analyze the previous/current radio conditions and communication paradigms in wireless communications, such as spectrum utilization, channel capacity, power level, antenna configurations, and heterogeneous link properties and help to generate an optimal action to improve the quality of service (QoS).

Recently, some machine learning algorithms have been proposed for wireless sensor networks, cognitive radio networks, bio-inspired networks, machine-to-machine communications, MIMO link adaption, antenna selection, congestion control, etc. Machine learning has been one of the most active research fields due to its great success in a wide range of domains. However, its impact on wireless communications has so far been very limited. The main challenge is how to formulate the problems in communication systems as a proper machine learning model.

The paper "Multilayer Learning Network for Modulation Classification Assisted with Frequency Offset Cancellation in Satellite to Ground Link" addresses the issue of carrier frequency offset. The frequency offset is canceled using multilayer learning framework, while the same learning framework is also used for modulation classification. The paper "Pedestrian Motion Learning Based Indoor WLAN Localization via Spatial Clustering" proposes a new pedestrian motion learning-based indoor wireless local area network localization approach. This paper achieves satisfactory locationbased services without the demand for location calibration or motion sensors. The paper "Distributed Routing Strategy Based on Machine Learning for LEO Satellite Network" focuses on effective and reliable routing for LEO satellite network. Considering the traffic distribution density on the surface of the earth, the extreme learning machine based distributed routing strategy makes routing decision based on traffic prediction. The paper "WiFi Positioning Based on User Orientation Estimation and Smartphone Carrying Position Recognition" proposes a novel WiFi fingerprinting positioning approach considering both user orientations using a variant of principle-component analysis and smartphone carrying positions by employing robust random forest classifier. Experimental results show that the proposed WiFi positioning approach may improve positioning accuracy significantly. The paper "The Parallel Algorithm Based on 
Genetic Algorithm for Improving the Performance of Cognitive Radio" focuses on the intercarrier interference (ICI) problem of cognitive radio (CR). In this paper, the machine learning algorithm is used to obtain the optimal interference subcarriers of an unlicensed user. Moreover, the parallel ICI suppression algorithm is designed to improve the calculation speed and meet the practical requirement of CR.

The set of five papers is carefully selected to present the readers with how different machine learning technologies, ranging from classical principal component analysis to multilayer learning networks, find their applications in different areas of wireless communications. In particular, the works presented in this special issue demonstrate machine learning applications across the communication protocol stack, from techniques of digital modulations, cognitive radio, and routing, to applications in positioning and motion learning.

\section{Conflicts of Interest}

The authors declare that there are no conflicts of interest regarding the publication of this paper.

\section{Acknowledgments}

The guest editors would like to thank all reviewers for their efforts in reviewing manuscripts submitted to this special issue. This special issue is partially supported by the National Natural Science Foundation of China under Grant No. U1733114 and No. 61631017 and the Fundamental Research Funds for the Central Universities under Grant No. 22120180089.

Xin-Lin Huang

$\mathrm{Fei} \mathrm{Hu}$

Xiaomin $\mathrm{Ma}$

Ioannis Krikidis

Dejan Vukobratovic 


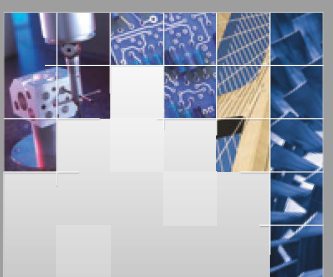

\section{Enfincering}
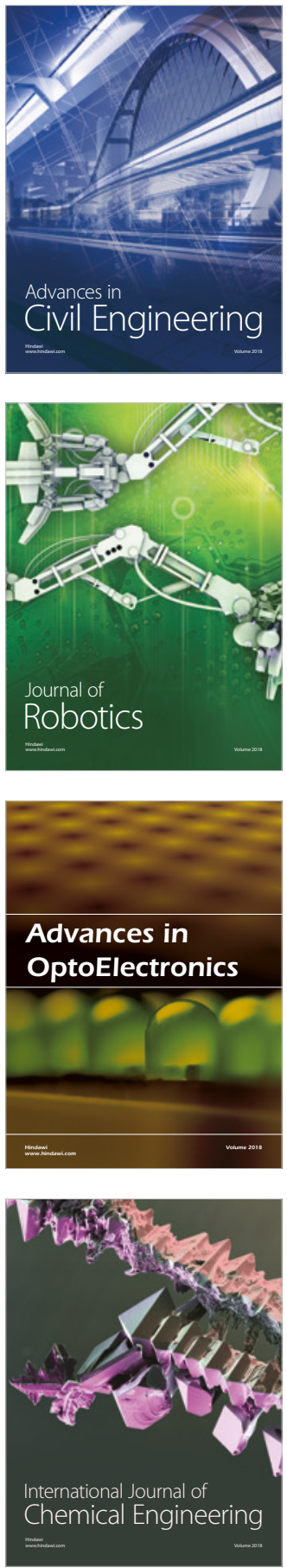

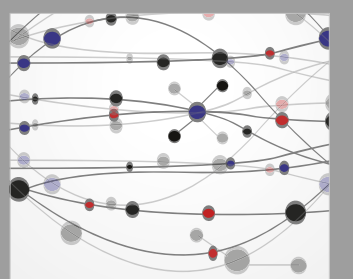

\section{Rotating \\ Machinery}

The Scientific World Journal

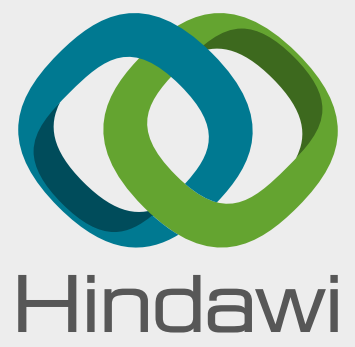

Submit your manuscripts at

www.hindawi.com
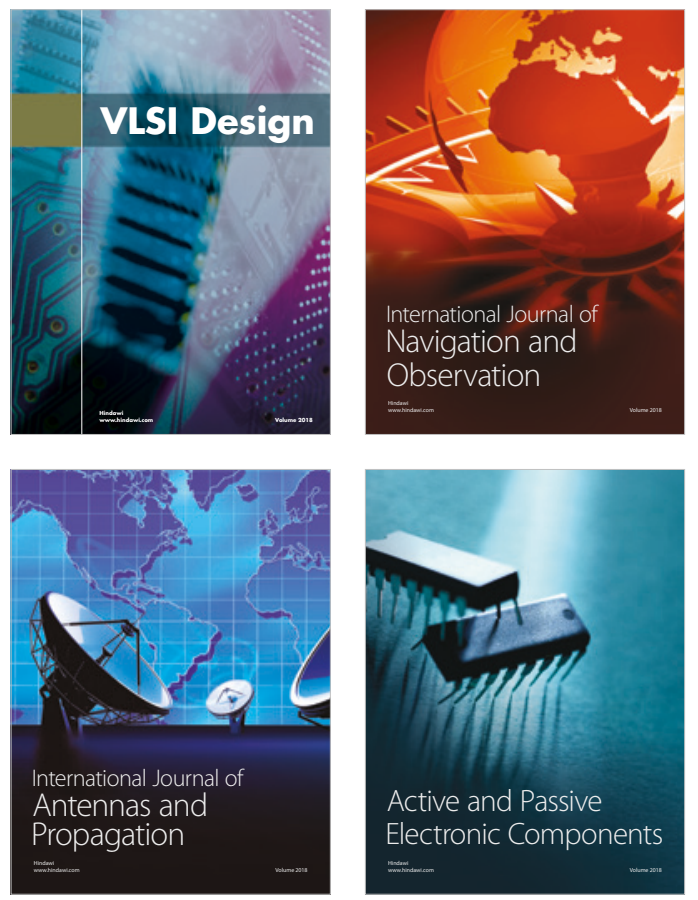
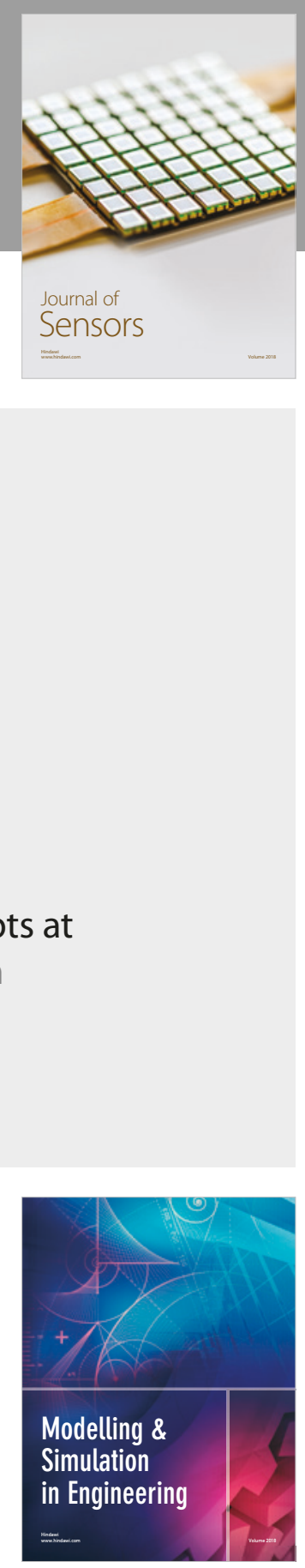

\section{Advances \\ Multimedia}
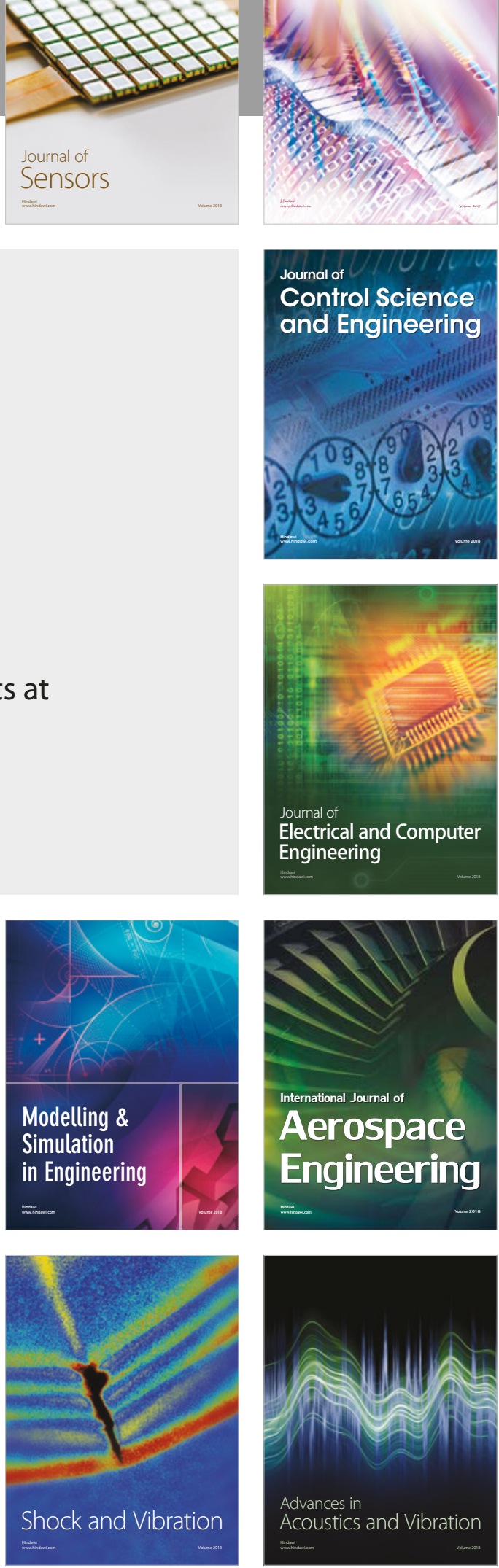